\title{
Stopping, goal-conflict, trait anxiety and frontal rhythmic power in the stop-signal task
}

\author{
Phoebe S.-H. Neo • Jane K. Thurlow • Neil McNaughton
}

Published online: 7 June 2011

(C) Psychonomic Society, Inc. 2011

\begin{abstract}
The medial right frontal cortex is implicated in fast stopping of an initiated motor action in the stop-signal task (SST). To assess whether this region is also involved in the slower behavioural inhibition induced by goal conflict, we tested for effects of goal conflict (when stop and go tendencies are balanced) on low-frequency rhythms in the SST. Stop trials were divided, according to the delays at which the stop signal occurred, into short-, intermediate-, and long-delay trials. Consistent with goal-conflict processing, intermediate-delay trials were associated with greater $7-8 \mathrm{~Hz}$ EEG power than short- or long-delay trials at medial right frontal sites (Fz, F4, and F8). At F8, 7-8 Hz power was linked to high trait anxiety and neuroticism. A separate $4-7 \mathrm{~Hz}$ power increase was also seen in stop, relative to go, trials, but this was independent of delay, was maximal at the central midline site $\mathrm{Cz}$, and predicted faster stopping. Together with previous data on the SST, these results suggest that the right frontal region could be involved in multiple inhibition mechanisms. We propose a hierarchical model of the control of stopping that integrates the literature on the neural control of fast motor stopping with that on slower, motive-directed behavioural inhibition.
\end{abstract}

Keywords EEG $\cdot$ Prefrontal cortex $\cdot$ Behavioural inhibition $\cdot$ Conflict $\cdot$ Trait anxiety $\cdot$ Stop-signal task

P.S.-H.N. was supported by a postgraduate scholarship from the Department of Psychology, University of Otago.

P. S.-H. Neo $(\bowtie) \cdot$ J. K. Thurlow $\cdot$ N. McNaughton

Department of Psychology, University of Otago,

P.O. Box 56, Dunedin 9054, New Zealand

e-mail: phoebe@psy.otago.ac.nz
Dysfunctional behavioural inhibition is implicated in many disorders: attention-deficit/hyperactivity disorder (Aron \& Poldrack, 2005), substance abuse (Fillmore \& Rush, 2002; Monterosso, Aron, Cordova, Xu, \& London, 2005), Parkinson's disease (Gauggel, Rieger, \& Feghoff, 2004), and anxiety disorders (Gray \& McNaughton, 2000). But "behavioural inhibition" is a descriptive term. It encompasses multiple paradigms and, potentially, multiple processes; it also appears to engage overlapping neural networks (Enriquez-Geppert, Konrad, Pantev, \& Huster, 2010; Rubia, Russell, Overmeyer, Brammer, Bullmore, \& Sharma, 2001). A challenge, then, is to identify and distinguish the processes engaged during behavioural inhibition.

The neural basis of stopping an initiated action is widely investigated with the stop-signal task (SST; Logan, Cowan, \& Davis, 1984). "Go" trials require a simple fast response to a "go" signal. On some ("stop") trials, a second signal is also presented after a variable delay, which requires inhibition of the ongoing go process. Stop trials, when contrasted with go trials, consistently activate medial right frontal cortical networks that can include the anterior cingulate cortex (ACC), right inferior frontal gyrus (rIFG), and presupplementary motor area (preSMA) (Aron, Fletcher, Bullmore, Sahakian, \& Robbins, 2003; Boehler, Appelbaum, Krebs, Hopf, \& Woldorff, 2010; Floden \& Stuss, 2006; Li, Yan, Bergquist, \& Sinha, 2007; Sharp, Bonnelle, De Boissezon, Beckmann, James, \& Patel, 2010). In particular, damage to the rIFG, but not the left IFG, has been shown to slow stop inhibition times (Aron et al., 2003).

This medial right frontal network may not be limited to simple, fast motor stopping. It could also mediate the motor 
inhibition resulting from motivational evaluation by the behavioural inhibition system (BIS; Gray \& McNaughton, 2000). When equally valued but incompatible goals compete for the control of behaviour, limbic structures in the BIS are thought to inhibit prepotent behaviour and initiate goal-conflict resolution. Application of the BIS theory to personality (Corr, 2008) has prompted psychometric development of the BIS personality scale (Carver \& White, 1994). Notably, this scale has been linked to variation in medial (Boksem, Tops, Wester, Meijman, \& Lorist, 2006) and right frontal regions. In particular, the BIS scale has been linked to the rIFG (Shackman, McMenamin, Maxwell, Greischar, \& Davidson, 2009).

The common link with the rIFG suggests that simple stopping and BIS output may be processed in similar frontal networks. However, the BIS scale was not neurally anchored during its development. It may tap into two distinct factors (Beck, Smits, Claes, Vandereycken, \& Bijttebier, 2009; Heym, Ferguson, \& Lawrence, 2008): not only the passive avoidance associated with the BIS, but also quite separate active avoidance processing (Perkins, Kemp, $\&$ Corr, 2007).

We, therefore, devised a direct test of the neural effects of goal conflict to confirm the BIS link with medial right frontal cortex suggested by the personality scale data. This used the same version of the SST task used by Aron and Poldrack (2006), but our analysis had three important additional features.

Firstly, we assessed variation in neural response with variation in the stop-signal delay. Stop-related activation in the prefrontal cortex is similar when stopping is successful and when it fails to prevent the go process (Boehler et al., 2010; Garavan, Ross, Murphy, Roche, \& Stein, 2002). Assuming, as in the horse race model (Logan et al., 1984), that the go and stop processes proceed largely independently, goal conflict should be strongest when the peaks of their separate activations tend to coincide and successful inhibition is close to $50 \%$ (i.e., at intermediate stop-signal delays). With shorter or longer delays, the peak of the go (or stop) activation will overlap one of the tails of the stop (or go) activation-allowing one or the other to win the race and generating less stop-go conflict.

Secondly, we tested for a relation between neural response in the intermediate-delay trials and anxietyrelated personality measures. Goal-conflict processing should be linked to aversive emotion, particularly anxiety (Gray \& McNaughton, 2000; Johnson, Turner, \& Iwata, 2003). Even in a superficially unthreatening task such as the SST, threat-sensitive individuals such as high traitanxiety and neuroticism scorers would be predicted to show enhanced activation relative to low scorers if the task involves goal conflict.
Thirdly, we measured low-frequency rhythms in the 4- to $12-\mathrm{Hz}$ band likely to be homologous to that typical of rodent hippocampal rhythmical slow activity (Gray \& McNaughton, 2000; Miller, 1991). A core element of the BIS theory is the mediation of goal-conflict resolution by multiple cycles of recursive hippocampal-cortical interaction that are functionally dependent on rhythmical slow activity. In humans, the theta $(4-7 \mathrm{~Hz})$ and alpha $(9-12 \mathrm{~Hz})$ bands are generally considered to be functionally separate (Sauseng \& Klimesch, 2008). However, depth recording in humans suggests that task-related human hippocampal slow waves could extend beyond $4-7 \mathrm{~Hz}$, into the 8- to $12-\mathrm{Hz}$ range (Ekstrom, Caplan, Ho, Shattuck, Fried, \& Kahana, 2005; Jacobs, Korolev, Caplan, Ekstrom, Litt, \& Baltuch, 2010; Miller, 1991). Note that volume conduction from the hippocampus to frontal cortical sites would not be expected in the present study. However, goal conflict should result in rhythmic output to frontal networks, and so entrain frontal theta rhythms that are coherent with hippocampal slow waves (Young \& McNaughton, 2008).

Consequently, to examine whether medial right frontal activations during behavioural inhibition include a component of goal conflict, we used the SST to test for stopspecific increases in EEG spectral power within the 4- to $12-\mathrm{Hz}$ range at $\mathrm{Fz}, \mathrm{F} 4$, and $\mathrm{F} 8$ in trials with intermediate delays, as compared to those with either short or long delays. If goal conflict was detected, we predicted that it would be processed as an aversive signal, and so individuals with high trait anxiety and/or neuroticism should show higher goal-conflict-specific EEG power.

\section{Method}

\section{Participants}

The participants were students recruited from Otago University. The experiment was run in two replications. In Replication 1, 9 male and 9 female students (mean age = 20.22 years, $S D=1.22$ ) were recruited from the university's "Student Job Search" programme. They were each paid \$20 for their participation. In Replication 2, 13 male and 11 female students (mean age $=22.67$ years, $S D=3.12$ ), who were enrolled in a biopsychology course, participated voluntarily as part of the course's practical class requirement. The participants reported no psychological treatment in the past year. One participant in Replication 2 was left-handed and so was excluded. With this exception, all participants considered themselves right-handed. The recruitment of participants and other experimental procedures described below were approved by the University of Otago Ethics Committee (approval number: DP 10/07). 
The stop-signal task

The SST was set up to be, as far as possible, identical to that used in Aron and Poldrack (2006), and the control program used was translated from computer code provided by Adam Aron. Participants were told that both going and stopping were equally important. On a go trial (see Fig. 1), a white fixation circle was presented in the centre of the screen against a black background. Then, $500 \mathrm{~ms}$ later, a white arrow appeared in the circle. Participants were asked to make a left/right mouse click as quickly as possible in response to the left/right arrow. On stop trials, the stop signal (a tone) was presented at variable delays after the arrow. Participants were to withhold clicking if they heard the tone. They were presented with 384 trials in total, with a rest break after every 128 trials. Because one stop trial was presented in every four trials (the order was controlled by a random number generator), each 128-trial block consisted of 32 stop trials and 96 go trials.

Within each 128-trial block, the stop-signal delays (SSDs) were systematically varied between trials. This was controlled using a staircase tracking system. There were four, parallel staircases, which started at different SSDs $(100,150,200$, and $250 \mathrm{~ms})$. The four staircases were pseudorandomly assigned to the stop trials using a rule that

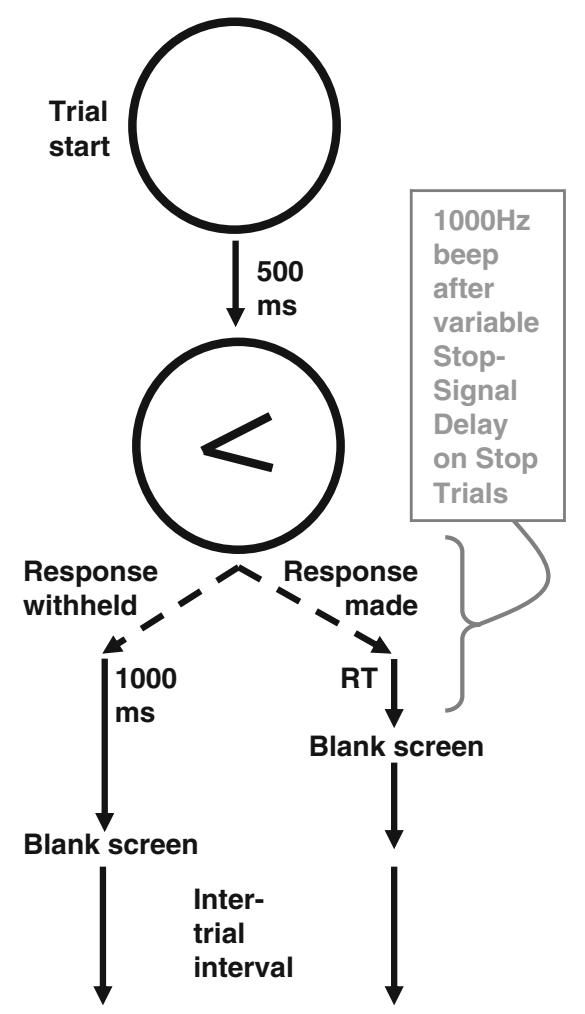

Fig. 1 Events in the stop-signal task ensured that each staircase moved eight times in each of the three 128-trial blocks. Failed inhibition in the current stop trial decreased the SSD, and successful inhibition increased the $\mathrm{SSD}$, by $50 \mathrm{~ms}$ apiece on the next trial that was controlled by that particular staircase.

Separating short-, intermediate-, and long-delay stop trials

The staircase procedure generated a tendency, on average, for responding to stabilise at a $50 \%$ probability of inhibition $\left(P_{\text {inhib }}\right)$ in the last 48 stop trials. We sorted these 48 trials by ascending SSDs and divided them into three groups (trials with the same SSD value were always included in the same group). Stop trials in each group were than averaged to give us short-, intermediate-, and longSSD trial groups. The number of trials in each average ranged from 8 to 21 trials.

\section{Data recording}

Participants were fitted with an Electro-Cap (ElectroCap International, USA). The data from electrodes F7, F3, Fz, F4, F8, T3, C3, Cz, C4, T4, T5, P3, Pz, P4, and T6 were recorded. Fp1 was used to detect ocular artefacts, and Gnd (anterior to Fz) was used as ground. Clip-on pure tin ear electrodes were linked together as reference. Amplification and recording were via a Mindset Model MS-1000 (Nolan Computer Systems, USA), with a bandpass of $1.8-36 \mathrm{~Hz}, 48 \mathrm{~dB} /$ octave roll-off, and a sample rate of $128 \mathrm{~Hz}$.

\section{EEG data reduction}

Residual mains noise was filtered using a three-point running mean (effective cutoff $43 \mathrm{~Hz}$ ). Ocular artefacts were corrected for automatically by fitting a template to the ballistic components recorded on Fp1 and then removing the fitted components from each channel via linear regression to leave residual EEG (Gratton, 1998). All remaining artefacts were removed manually by deletion of the same time segments across all channels and replacement of the data with missing values.

A 1-s overlapping Hanning window was applied to all trials analysed. For stop trials, the main attenuation of the window was in the $0.25 \mathrm{~s}$ leading and the $0.25 \mathrm{~s}$ trailing the $0.5-\mathrm{s}$ duration of the stop signal. The main power in the transform was derived for the 0.5 -s duration of the stop signal. For go trials, the window centred on the 0.5 -s duration at which the tone had been presented in the adjacent stop trial. The data were then fast Fourier transformed (frequency resolution of $1 \mathrm{~Hz}$ ). The Fourier-transformed data were then log transformed to normalise error variance, and averaged across trials. 
The 5 participants with more than $10 \%$ of their data deleted due to artefacts were excluded.

\section{Questionnaires}

The Eysenck Personality Questionnaire-Revised (EPQ-R) (Hodder \& Stoughton, U.K.) and the Spielberger State-Trait Anxiety Inventory (STAI) (Mind Garden Inc, CA) were also administered. These questionnaires were chosen as being well-validated measures of emotion and the predisposition to threat-related mental disorders, as opposed to the BIS scale, which is more focussed on behavioural inhibition and has not been demonstrated to be a predictor of disorder.

\section{Procedure}

Consent forms, the EPQ-R, and the STAI-Trait were administered upon participants' arrival (in Replication 2, participants had filled these out before arrival). The participants were then prepared for EEG recording. Electrode impedances were lowered to below $5 \mathrm{~K} \Omega$. Eyeblinks and relaxation-induced alpha rhythm were assessed to screen for oddities in the recordings. The STAI-State questionnaire was then administered, followed by behavioural testing. The STAI-State was administered again immediately after testing.

\section{Data analysis}

The ANOVAs were performed with the PASW Statistics 18 package. We tested for the predicted effects of goal conflict (an increase in spectral power in the intermediate-SSD, relative to short- and long-SSD, trials within the 4- to $12-\mathrm{Hz}$ frequency range) at the medial right frontal sites Fz, F4, and F8. Effects specific to stopping were assessed as the difference in power between stop and go trials (stop-go) in the 0.5 -s duration of the stop signal. The effects of SSD were assessed via orthogonal linear and quadratic contrasts (Snedecor \& Cochran, 1967) with the short-, intermediate-, and longSSD trials as successive value levels. Mathematically, in this three-level case, the linear contrast was the difference between the long- and short-SSD trials, with no contribution from the intermediate ones. The quadratic contrast was the difference between the intermediate and the average of the short and long trials. The contrasts did not, therefore, assume actual underlying linear or quadratic functions. Any frequency changes across 4-12 $\mathrm{Hz}$ were also assessed with linear and quadratic contrasts with $4,5,6,7,8,9,10,11$, and $12 \mathrm{~Hz}$ as successive values. Changes across recording sites were assessed with linear and quadratic contrasts with Fz, F4, and F8 as successive values. The initial EEG analyses detected no differences between the two replications, so only combined EEG and behavioural results are presented below. All $p$ values reported are uncorrected unless stated otherwise.

\section{Results}

\section{Behavioural measures}

The median recorded reaction time (RT) in the go trials, averaged across the two replications, was $448 \mathrm{~ms}(S D=91$; the mouse key was polled every $8 \mathrm{~ms}$, so the true average go RT was $4 \mathrm{~ms}$ less than the recorded value). The average stop-signal reaction time (SSRT) was $202 \mathrm{~ms}(S D=59)$. This was computed by subtracting a participant's average SSD (calculated from the last 48 stop trials) from their median go RT (in milliseconds) for correct go trials. The average SSD for Staircases 1 and 2 was $246 \mathrm{~ms}(S D=135)$, and that for Staircases 3 and 4 was $258 \mathrm{~ms}(S D=150)$. These values are all comparable to those arrived at by Aron and Poldrack (2006). We achieved $50 \% P_{\text {inhib }}$ stability in the last 48 stop trials. These were further separated according to their SSDs. The intermediate-SSD trials produced $50 \% P_{\text {inhib }}$; short-SSD trials, $75 \% P_{\text {inhib }}$; and long-SSD trials, $27 \% P_{\text {inhib. }}$. The percentage correct on go trials was $95 \%$ on average.

\section{Quadratic effect of SSDs}

We did not detect significant effects that varied linearly with SSD. However, as predicted, we detected power differences consistent with the presence of goal conflict. Stop-go power was significantly greater in the intermediate-SSD trials than in the adjacent short- and long-SSD trials. As can be seen in Fig. 2a, this effect was greatest at 7 and $8 \mathrm{~Hz}$ and was not significantly different across recording sites Fz, F4, and F8 [Stop-Go $\times$ Quadratic of SSD $\times$ Quadratic of Frequency: $F(1,35)=16.38, p<.001]$. As shown in the Fig. 2a insets, average $7-8 \mathrm{~Hz}$ power was indeed greater in the intermediate-SSD trials as compared to both the short- and long-SSD trials. The quadratic is not a mathematical artefact of a large difference between the intermediate condition and just one of the adjacent conditions.

We inspected the remaining recording sites $-\mathrm{F} 7, \mathrm{~F} 3$, T3, C3, Cz, C4, T4, T5, P3, Pz, P4, and T6-for similar SSD effects. The left frontal site, F3, adjacent to Fz, showed signs of an increase in $7-8 \mathrm{~Hz}$ power in the intermediate-SSD trials, but this increase was smaller than that at Fz, and there was no sign of a similar effect at the adjacent left frontal site F7. A post-hoc analysis of all five frontal sites showed that the effect of SSD at 7-8 $\mathrm{Hz}$ tended to increase from left to right [Stop-Go $\times$ Quadratic of SSD $\times$ Linear of Sites: $F(1,35)=10.279$, $p<.003]$. There was no evidence of quadratic SSD effects in the remaining nonfrontal sites. 
a Quadratic coefficient of SSD power variation
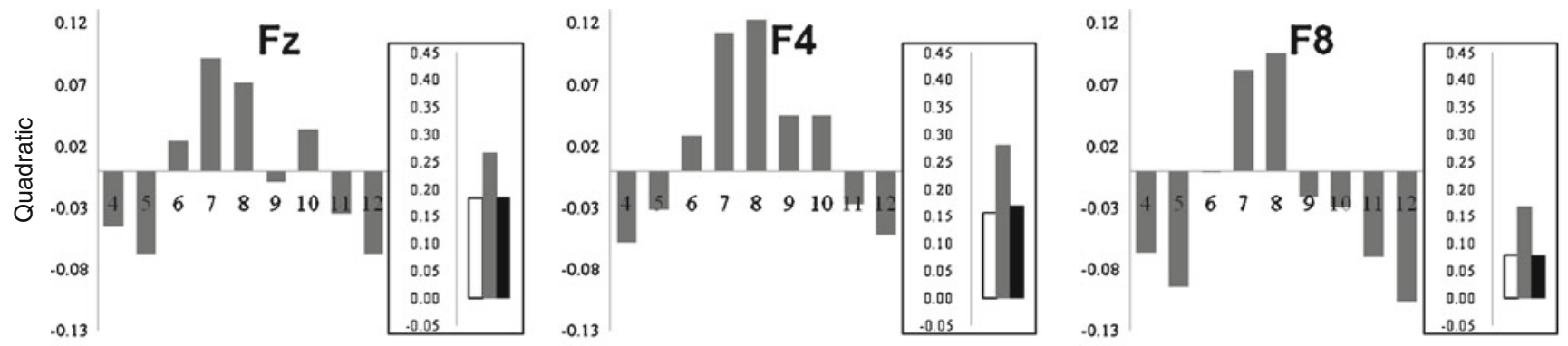

b Power averaged across all SSDs
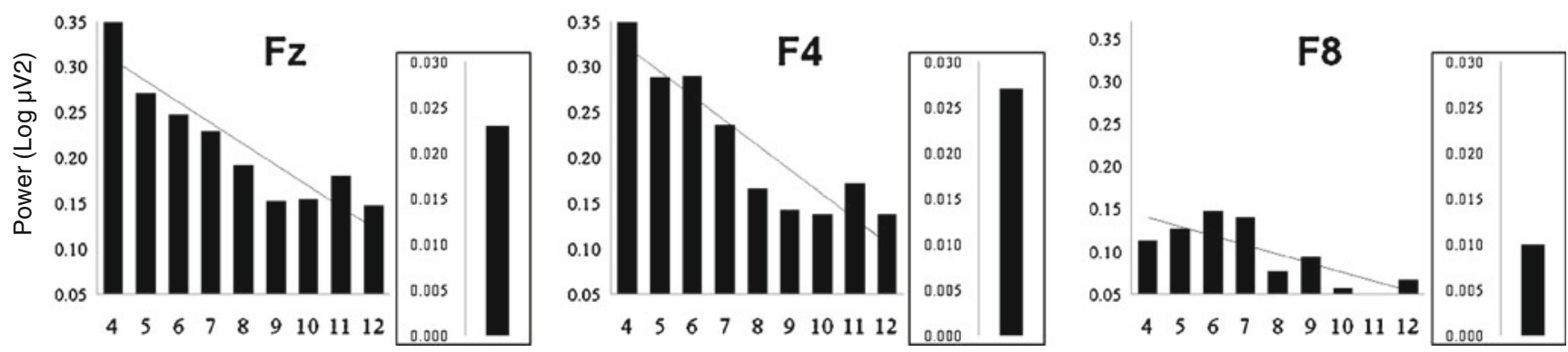

Frequency

Fig. 2 Power in stop trials relative to go trials. (a) Frequency variation in goal-conflict-specific power, estimated as the difference between power in the intermediate-SSD trials and the average power of the short- and long-SSD trials (quadratic). Inset: Average 7-8 Hz power for trials with short (white), intermediate (grey), and long (black) delay. (b) Frequency variation in average power across short-, intermediate-, and long-delay trials. Inset: The size of the linear coefficient of the fitted power frequency functions
Quadratic effect of SSDs, SSRT, and threat-related personality traits

To further characterise the intermediate-SSD power, we calculated stop-go 7-8 $\mathrm{Hz}$ power in the intermediate-SSD trials for Fz, F4, and F8. These were entered as predictor variables to predict SSRT in a stepwise regression. We estimated SSRT following the formula used previously (Aron \& Poldrack, 2006), according to the assumptions of the horse race model. Reliable relationships were not detected.

Next, we assessed whether this intermediate-SSD power predicted threat-related personality traits. The values for 7-8 $\mathrm{Hz}$ intermediate-SSD power at $\mathrm{Fz}, \mathrm{F} 4$, and $\mathrm{F} 8$ were entered as predictor variables in separate stepwise regressions to predict trait anxiety and neuroticism, respectively. High 7-8 Hz intermediate-SSD power at F8 emerged as the single best predictor of high trait-anxiety scores $\left[r^{2}=.126\right.$; $F(1,34)=4.92, p<.033$; Fig. 3a] and, in a separate stepwise regression, of high neuroticism scores $\left[r^{2}=.112\right.$; $F(1,34)=4.279, p<.046$; Fig. 3b]. As shown in Table 1, no other simple correlations with the personality traits were evident.
We then forced trait-anxiety and neuroticism scores into the same equation to predict the $7-8 \mathrm{~Hz}$ power at F8, in order to explore the variance structure. The variance of $7-8 \mathrm{~Hz}$ power accounted for by neuroticism uniquely was close to zero, and that of trait anxiety was negligible at about $2 \%$. Neuroticism and trait anxiety had a shared predictive power of slightly over $10 \%$. The analysis above should be seen as exploratory rather than evidential, given the small sample size and marginal $p$ value of the F8-personality traits' effects (which is relatively weak, as compared to the other effects). However, the results are consistent with the changes in $7-8 \mathrm{~Hz}$ power at $\mathrm{F} 8$ being linked to the shared variance between trait anxiety and neuroticism.

\section{Average SSD effects}

Previous SST studies generally tested for the effects of stopping averaged across trials with different SSDs. To facilitate comparisons across studies, we also tested for similar effects of stopping. We detected at Fz, F4, and F8 overall stop-go effects that did not vary with SSD. These were at different frequencies from the quadratic effects of 

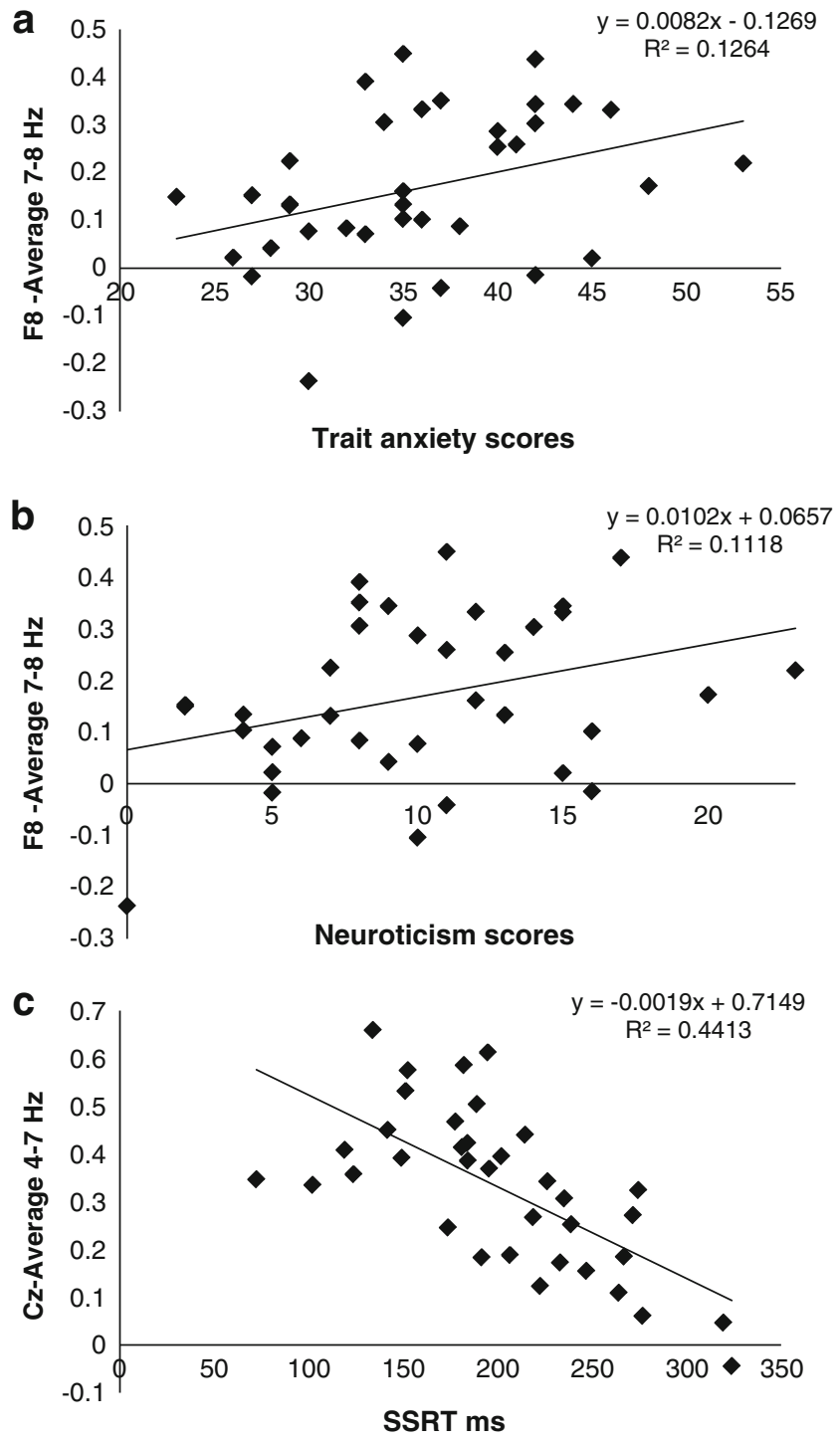

Fig. 3 (a) Correlation between trait anxiety scores and 7-8 Hz power in the intermediate-delay trials at F8. (b) Correlation between neuroticism scores and 7-8 Hz power in the intermediate-delay trials at F8. (c) Correlation between average $4-7 \mathrm{~Hz}$ power (all delay trials) at $\mathrm{Cz}$ (note the different scale from panels $\mathrm{A}$ and $\mathrm{B}$ ) and stop-signal reaction times (SSRT)

Table 1 Correlation matrix of $7-8 \mathrm{~Hz}$ intermediate SSD power (at electrode sites Fz, F4, F8), trait anxiety (TA), and neuroticism (N)

Pearson correlations / (Significance, 1-tailed)

\begin{tabular}{llllll}
\hline & TA & N & Fz & F4 & F8 \\
\hline TA & & .000 & .313 & .197 & .017 \\
$\mathrm{~N}$ & .826 & & .157 & .105 & .023 \\
$\mathrm{Fz}$ & .084 & .173 & & .000 & .381 \\
$\mathrm{~F} 4$ & .146 & .214 & .903 & & .126 \\
$\mathrm{~F} 8$ & .356 & .334 & -.052 & .196 & \\
\hline
\end{tabular}

SSDs. As is shown in Fig. 2b, these effects were greater at the lower frequencies $(4-7 \mathrm{~Hz}$ ) and smaller at the higher frequencies $(8-12 \mathrm{~Hz})($ Stop-Go $\times$ Linear of Frequency: $F(1,35)=26.08, p<.001)$. However, as shown in the Fig. $2 b$ insets, the size of the linear trend of frequency varied between recording sites, with the largest difference observed at F4, followed by Fz and then F8 (Stop-Go $\times$ Linear of Frequency $\times$ Quadratic of Sites: $F(1,35)=8.4, p<.006)$.

When we inspected the remaining sites-F7, F3, T3, C3, $\mathrm{Cz}, \mathrm{C} 4, \mathrm{~T} 4, \mathrm{~T} 5, \mathrm{P} 3, \mathrm{Pz}, \mathrm{P} 4$, and T6-for similar effects, we found that this average SSD stop-go effect at $4-7 \mathrm{~Hz}$ was evident across all of the recording sites [Stop-Go: $F(1,35)=$ 162.6, $p<.001]$. However, the size of the effect appeared to vary. $\mathrm{Cz}$ and $\mathrm{P} 4$ showed the biggest effects, followed by $\mathrm{F} 4$, $\mathrm{P} 3$, and $\mathrm{Fz}$, respectively. The variation in the effect size was assessed statistically by dividing the 15 recording sites into a $3 \times 5$ matrix. The electrodes in the front, middle, and back rows were consecutive levels of the factor Front-Back, and the electrodes in the far left, left, midline, right, and far right columns were consecutive levels of the factor Left-Right. This demonstrated significant, substantially centralised, topographic variance [Stop-Go $\times$ Quadratic of Front-Back $\times$ Quadratic of Left-Right: $F(1,35)=8.927, p<.005$; StopGo $\times$ Quadratic of Front-Back $\times$ Linear of Left-Right: $F(1,35)=4.894, p<.034$; Stop-Go $\times$ Linear of FrontBack $\times$ Quadratic of Left-Right: $F(1,35)=5.114, p<.03$ ]

Average SSD power, SSRT, and threat-related personality traits

We repeated the stepwise procedure as per our goal-conflict analyses, replacing 7-8 Hz intermediate-SSD power (at Fz, F4, and F8) with average $4-7 \mathrm{~Hz}$ power as a predictor variable of SSRT, trait anxiety, and neuroticism, respectively. The 4 $7 \mathrm{~Hz}$ power at Fz emerged as the single best predictor of faster SSRT $\left[r^{2}=.35, F(1,34)=18.496, p<.001\right]$. We did not detect any reliable effects with the personality traits.

Given that we had no a priori predictions in relation to this 4-7 Hz effect, and given that it was evident across all of the recording sites, we created a correlation matrix of its link with SSRT across all recording sites to further characterise its properties. As is shown in Table 2, Cz, which is just posterior to $\mathrm{Fz}$, showed the largest $r$ value (Fig. 3c). As an exploratory step, we forced $\mathrm{Fz}$ and $\mathrm{Cz}$ power into the same equation to predict SSRT. Cz accounted for all of the predictive power of $\mathrm{Fz}$ in relation to SSRT, and was additionally associated with about $9 \%$ unique predictive power.

\section{Discussion}

We observed a medial right frontal (Fz, F4, F8) increase in spectral power at $7-8 \mathrm{~Hz}$ to the stop signal (as compared to 
Table 2 Correlation matrix of average 4-7 Hz SSD power at all sites analysed and SSRT

\begin{tabular}{lccccc}
\hline & F7 & F3 & Fz & F4 & $\mathrm{F} 8$ \\
\hline SSRT & -.164 & -.609 & -.593 & -.502 & -.109 \\
$P(1$-tailed $)$ & .169 & .000 & .000 & .001 & .263 \\
& $\mathrm{~T} 3$ & $\mathrm{C} 3$ & $\mathrm{Cz}$ & $\mathrm{C} 4$ & $\mathrm{~T} 4$ \\
SSRT & -.270 & -.562 & -.664 & -.543 & -.297 \\
$P(1$-tailed $)$ & .056 & .000 & .000 & .000 & .039 \\
& $\mathrm{~T} 5$ & $\mathrm{P} 3$ & $\mathrm{Pz}$ & $\mathrm{P} 4$ & $\mathrm{~T} 6$ \\
SSRT & -.370 & -.493 & -.525 & -.586 & -.311 \\
$P(1$-tailed $)$ & .013 & .001 & .001 & .000 & .032 \\
\hline
\end{tabular}

a

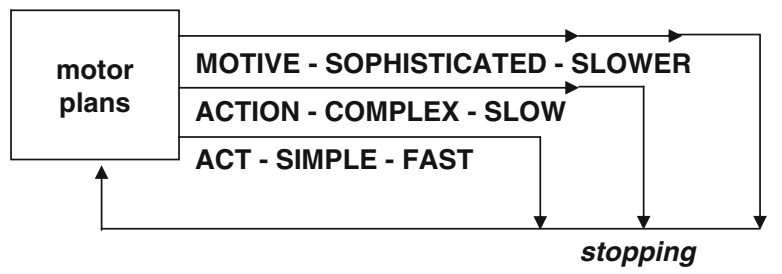

b

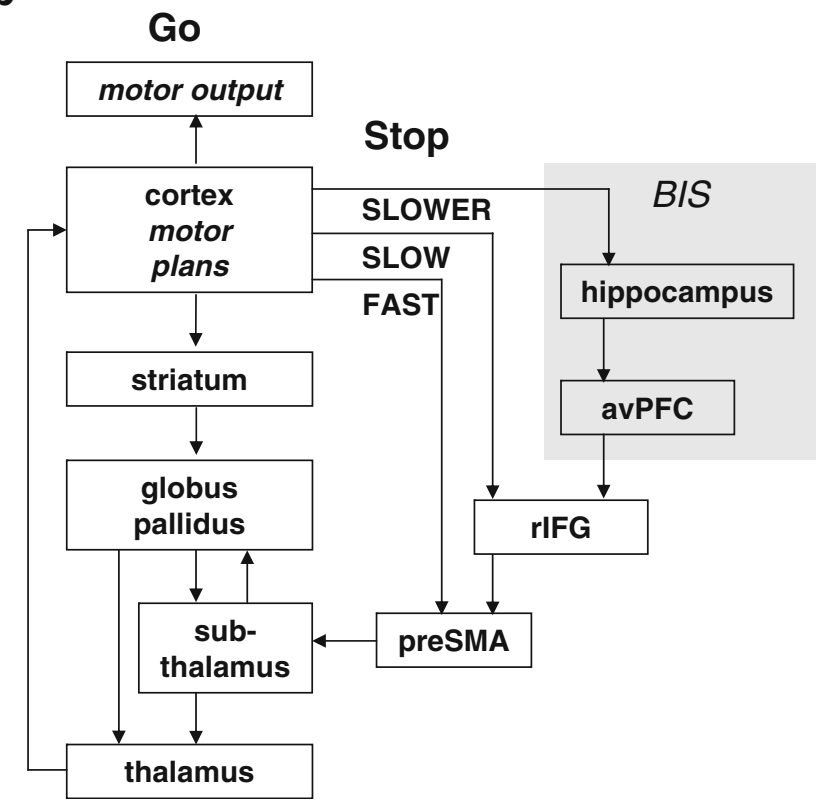

Fig. 4 Postulated neural control of going and stopping. Motor inhibition uses both fast and slow routes (Nachev, Kennard, \& Husain, 2008) to modulate the go circuit (Nachev, Rees, Parton, Kennard, \& Husain, 2005; Nachev, Wydell, O’Neill, Husain, \& Kennard, 2007). We propose that goal inhibition involves, in addition, the slower BIS circuit (Gray \& McNaughton, 2000). avPFC = anteroventral prefrontal cortex; $\mathrm{rIFG}=$ right inferior frontal gyrus; preSMA $=$ presupplementary motor area. Connections have been simplified, and circuits and structures in the BIS, other than the hippocampus, are not shown (e.g., the Papez circuit is omitted) the matching point in go trials). This is greatest in the intermediate-delay trials, relative to the average of the short- and long-delay trials, and is consistent with the predicted effects of goal conflict on the different stop-signal delay trials. Similar effects were not detected at other recording sites.

Efficient and accurate information processing in the BIS requires recursive processing - that is, signals carrying information circulate the relevant network for multiple cycles (see Gray \& McNaughton, 2000, pp. 24-27). SSRT was approximately $200 \mathrm{~ms}$ in the present study. It takes about $430 \mathrm{~ms}$ to complete three cycles of a $7-\mathrm{Hz}$ rhythm. If the 7-8 Hz intermediate-delay rhythmic power observed here represented even as few as three recursive cycles of goal-conflict processing by the BIS, it would have continued after stopping was generated by at least $230 \mathrm{~ms}$ - probably too late to affect inhibition. This could explain the lack of a correlation between the 7-8 Hz power and SSRT. This suggests that a simple motor inhibition task like the SST, which is expected to recruit relatively fast motor processing, may also activate slower and/or longer lasting recursive motivational processes induced by goal conflict, which would have increased behavioural inhibition in tasks involving slower responses.

The apparent links between high 7-8 Hz intermediatedelay power at the right frontal site F8 and high trait anxiety and neuroticism also support the activation of a motivational component of goal conflict by the SST. Although small (10\% of the variance) and statistically weak ( $\sim \%$ without Bonferroni correction), these links are consistent with the interpretation by the BIS theory of goal conflict as activating a class of aversive mechanisms. Allowing for differences in paradigms and measures, our results are also broadly consistent with previous studies linking a personality factor of the BIS to medial right frontal processing (Boksem et al., 2006; Shackman et al., 2009; Wacker, Chavanon, Leue, \& Stemmler, 2010).

Taken together, our results suggest that in addition to simple motor inhibition, previous right frontal SST activations (Aron et al., 2003; Boehler et al., 2010; Li et al., 2007; Savostyanov et al., 2009; Sharp et al., 2010) could include a motivational mechanism induced by goal conflict.

Note that the 7-8 Hz power at Fz appears distinct from that at F8, since it did not appear to show any relation to threat-related personality scores. Given our use of an effective 500-ms window and given an average SSRT of $200 \mathrm{~ms}$, the $\mathrm{Fz}$ rhythmic power could reflect outcome/ response conflict processing of the type specified in the response conflict monitoring theory (Botvinick, 2007). The conflict monitoring theory implicates midline structures such as the anterior cingulate in the evaluation of discrepancies between actual and expected outcomes. On this view, 
the linking of the BIS scale to error-related negativity (Boksem et al., 2006), which has been linked to the anterior cingulate (Yeung, Botvinick, \& Cohen, 2004), could potentially be the result of the inclusion in that scale of items related to active avoidance rather than goal conflict (Beck et al., 2009; Heym et al., 2008).

Relative to go responses, stop responses were accompanied by increased 4-7 Hz power. This power differed from the concurrently recorded $7-8 \mathrm{~Hz}$ effects not only in frequency, but also in that it did not vary across short-, intermediate- and long-delay trials. A further difference is that it was observed to varying extents across all of the recording sites and showed the greatest power, and the best SSRT predictor, at $\mathrm{Cz}$. The SST is thought to be controlled by two parallel networks involving the rIFG and preSMA/SMA, respectively. These show different levels of participation in action inhibition, depending on the speed of the go reaction times. When go times are particularly fast, rIFG activations occur but appear to be insufficiently fast to influence inhibition-control is exercised, instead, by preSMA/SMA (Aron et al., 2003; Floden \& Stuss, 2006; Garavan et al., 2002). Under normal, less urgent, circumstances, both rIFG and preSMA appear to be concurrently involved in the SST (Duann, Ide, Luo, \& Li, 2009; Floden \& Stuss, 2006; Garavan et al., 2002). Our present methods do not allow for source localisation, but given that our go times were relatively fast as compared to patient groups (Aron et al., 2003; Floden \& Stuss, 2006), the correlation of $\mathrm{Cz}$ power with SSRT is more compatible with greater involvement of central frontal fast (preSMA/SMA) control of stopping (Carretie, Tapia, Mercado, Albert, LopezMartin, \& de la Serna, 2004) than with slower (rIFG) control.

The detection of $7-8 \mathrm{~Hz}$ rhythmic power at F8 that is consistent with the effects of goal conflict, the link of this power with trait anxiety and neuroticism, and the lack of a link with SSRT (in contrast to $\mathrm{Cz}$ power) lead us to speculate that the neural elements of the BIS (Gray \& McNaughton, 2000) could form a third, SLOWER circuit, parallel to the FAST and SLOW circuits previously demonstrated (Fig. 4a). We hypothesise that this circuit relays its outputs to the motor system via the right frontal region, possibly the rIFG. Not only has the rIFG been consistently demonstrated to play a role in action stopping (Aron et al., 2003; Chambers, Bellgrove, Stokes, Henderson, Garavan, \& Robertson, 2006; Chevrier, Noseworthy, \& Schachar, 2007), it has also been linked to BIS-scale-related alpha suppression (Shackman et al., 2009). It is a likely target for the slower-to-arrive output from the BIS, which would involve a parallel return loop, with information passing from the motor planning areas directly or indirectly to the hippocampus, and then back to areas controlling stopping. Moreover, the hippocampus has direct connections to anterior ventral prefrontal cortex, which has direct connections to rIFG (see Figure 1.9 in Gray \& McNaughton, 2000). We propose a model, therefore, in which the BIS (shaded areas in Fig. 4b) operates in parallel with, and relays through, rIFG in the same way that rIFG has been shown to be in parallel with, and to relay through, preSMA.

In conclusion, stopping could involve a slower, recursive, motive-directed "goal"-conflict process, superimposed on a slow "action" process, which is superimposed on a fast "act" process (Fig. 4). Such a hierarchical system would allow adaptive selection between "quick and dirty" and "slow and sophisticated" decision/response mechanisms, depending on the time pressures imposed on the go process. Due to the limits of source localisation, our present results have suggested but do not provide any direct evidence for this hierarchical neural model. It should also be noted that while we have mapped $4-12 \mathrm{~Hz}$ rhythms to the SLOWER (BIS) circuit, this is based on extensive previous work on the BIS in rodents, and not on the present data. Whether higher-frequency rhythmic activity is important for the SLOW and FAST circuits is unclear. To our knowledge, a mapping of specific rhythmic activity to the respective circuits has not been examined, or postulated. Evidence for them in the present model is based on fMRI and lesions (Aron et al., 2003; Floden \& Stuss, 2006), which do not provide evidence in relation to rhythmicity.

While we have recorded changes in rhythmic power (at F8) consistent with the effect of goal conflict predicted from BIS theory over areas associated with stopping, there is no direct evidence that this was BIS activation. Our model is, therefore, a speculative amalgam of the two detailed neurologies previously described for the BIS and the stopping system. The model is falsifiable, however, and we see it as providing a basis for further work leading to an integrated approach to the multiple disorders in which behavioural inhibition has been implicated (Fillmore \& Rush, 2002; Gauggel et al., 2004; Gray \& McNaughton, 2000; Monterosso et al., 2005).

\section{References}

Aron, A. R., Fletcher, P. C., Bullmore, E. T., Sahakian, B. J., \& Robbins, T. W. (2003). Stop-signal inhibition disrupted by damage to right inferior frontal gyrus in humans. Nature Neuroscience, 6, 115-116.

Aron, A. R., \& Poldrack, R. A. (2005). The cognitive neuroscience of response inhibition: relevance for genetic research in attention-deficit/hyperactivity disorder. Biological Psychiatry, 57, 1285-1292.

Aron, A. R., \& Poldrack, R. A. (2006). Cortical and subcortical contributions to stop signal response inhibition: role of the subthalamic nucleus. Journal of Neuroscience, 26, 2424-2433.

Beck, I., Smits, D. J. M., Claes, L., Vandereycken, W., \& Bijttebier, P. (2009). Psychometric evaluation of the behavioral inhibition/ behavioral activation system scales and the sensitivity to punishment and sensitivity to reward questionnaire in a sample 
of eating disordered patients. Personality and Individual Differences, 47, 407-412. doi:10.1016/j.paid.2009.04.007.

Boehler, C. N., Appelbaum, L. G., Krebs, R. M., Hopf, J. M., \& Woldorff, M. G. (2010). Pinning down response inhibition in the brain-Conjunction analyses of the stop-signal task. NeuroImage, 52, 1621-1632. doi:10.1016/j.neuroimage.2010.04.276.

Boksem, M. A. S., Tops, M., Wester, A. E., Meijman, T. F., \& Lorist, M. M. (2006). Error-related ERP components and individual differences in punishment and reward sensitivity. Brain Research, 1101, 92-101. doi:10.1016/j.brainres.2006.05.004.

Botvinick, M. (2007). Conflict monitoring and decision making: reconciling two perspectives on anterior cingulate function. Cognitive, Affective, \& Behavioral Neuroscience, 7, 356-366.

Carretie, L., Tapia, M., Mercado, F., Albert, J., Lopez-Martin, S., \& de la Serna, J. M. (2004). Voltage-based versus factor score-based source localization analyses of electrophysiological brain activity: a comparison. Brain Topography, 17, 109-115.

Carver, C. S., \& White, T. L. (1994). Behavioral inhibition, behavioral activation, and affective responses to impending reward and punishment: the BIS/BAS scales. Journal of Personality and Social Psychology, 67, 319-333.

Chambers, C. D., Bellgrove, M. A., Stokes, M. G., Henderson, T. R., Garavan, H., Robertson, I. H., ... Mattingley, J. B. (2006). Executive "brake failure" following deactivation of human frontal lobe. Journal of Cognitive Neuroscience, 18, 444-455.

Chevrier, A. D., Noseworthy, M. D., \& Schachar, R. (2007). Dissociation of response inhibition and performance monitoring in the stop signal task using event-related fMRI. Human Brain Mapping, 28, 1347-1358.

Corr, P. J. (Ed.). (2008). The reinforcement sensitivity theory of personality. Cambridge: Cambridge University Press.

Duann, J. R., Ide, J. S., Luo, X., \& Li, C. R. (2009). Functional connectivity delineates distinct roles of the inferior frontal cortex and presupplementary motor area in stop signal inhibition. Journal of Neuroscience, 29, 10171-10179. doi:10.1523/jneur osci.1300-09.2009.

Ekstrom, A. D., Caplan, J. B., Ho, E., Shattuck, K., Fried, I., \& Kahana, M. J. (2005). Human hippocampal theta activity during virtual navigation. Hippocampus, 15, 881-889.

Enriquez-Geppert, S., Konrad, C., Pantev, C., \& Huster, R. J. (2010). Conflict and inhibition differentially affect the N200/P300 complex in a combined go/nogo and stop-signal task. NeuroImage, 51, 877-887. doi:10.1016/j.neuroimage.2010.02.043.

Fillmore, M. T., \& Rush, C. R. (2002). Impaired inhibitory control of behavior in chronic cocaine users. Drug and Alcohol Dependence, 66, 265-273.

Floden, D., \& Stuss, D. T. (2006). Inhibitory control is slowed in patients with right superior medial frontal damage. Journal of Cognitive Neuroscience, 18, 1843-1849.

Garavan, H., Ross, T. J., Murphy, K., Roche, R. A. P., \& Stein, E. A. (2002). Dissociable executive functions in the dynamic control of behavior: inhibition, error detection, and correction. NeuroImage, 17, 1820-1829.

Gauggel, S., Rieger, M., \& Feghoff, T. A. (2004). Inhibition of ongoing responses in patients with Parkinson's disease. Journal of Neurology, Neurosurgery, and Psychiatry, 75, 539-544.

Gratton, G. (1998). Dealing with artifacts: the EOG contamination of the event-related brain potential. Behavior Research Methods, Instruments, \& Computers, 30, 44-53.

Gray, J. A., \& McNaughton, N. (2000). The neuropsychology of anxiety: An enquiry into the functions of the septo-hippocampal system (2nd ed.). Oxford: Oxford University Press.

Heym, N., Ferguson, E., \& Lawrence, C. (2008). An evaluation of the relationship between Gray's revised RST and Eysenck's PEN: distinguishing BIS and FFFS in Carver and White's BIS/BAS scales. Personality and Individual Differences, 45, 709-715. doi:10.1016/j.paid.2008.07.013.

Jacobs, J., Korolev, I. O., Caplan, J. B., Ekstrom, A. D., Litt, B., Baltuch, G., ... Kahana, M. J. (2010). Right-lateralized brain oscillations in human spatial navigation. Journal of Cognitive Neuroscience, 22, 824-836.

Johnson, S. L., Turner, R. J., \& Iwata, N. (2003). BIS/BAS levels and psychiatric disorder: an epidemiological study. Journal of Psychopathology and Behavioral Assessment, 25, 25-36.

Li, C. S. R., Yan, P., Bergquist, K. L., \& Sinha, R. (2007). Greater activation of the "default" brain regions predicts stop signal errors. Neurolmage, 38, 640-648.

Logan, G. D., Cowan, W. B., \& Davis, K. A. (1984). On the ability to inhibit simple and choice reaction-time responses-A model and a method. Journal of Experimental Psychology. Human Perception and Performance, 10, 276-291.

Miller, R. (1991). Cortico-hippocampal interplay and the representation of contexts in the brain (Vol. 17). Berlin: Springer.

Monterosso, J. R., Aron, A. R., Cordova, X., Xu, J. S., \& London, E. D. (2005). Deficits in response inhibition associated with chronic methamphetamine abuse. Drug and Alcohol Dependence, 79, 273-277. doi:10.1016/j.drugalcdep.2005.02.002.

Nachev, P., Kennard, C., \& Husain, M. (2008). Functional role of the supplementary and pre-supplementary motor areas. Nature Reviews Neuroscience, 9, 856-869. doi:10.1038/nrn2478.

Nachev, P., Rees, G., Parton, A., Kennard, C., \& Husain, M. (2005). Volition and conflict in human medial frontal cortex. Current Biology, 15, 122-128. doi:10.1016/j.cub.2005.01.006.

Nachev, P., Wydell, H., O’Neill, K., Husain, M., \& Kennard, C. (2007). The role of the pre-supplementary motor area in the control of action. NeuroImage, 36, T155-T163. doi:10.1016/j. neuroimage.2007.03.034.

Perkins, A. M., Kemp, S. E., \& Corr, P. J. (2007). Fear and anxiety as separable emotions: an investigation of the revised reinforcement sensitivity theory of personality. Emotion, 7, 252-261.

Rubia, K., Russell, T., Overmeyer, S., Brammer, M. J., Bullmore, E. T., Sharma, T., ... Taylor, E. (2001). Mapping motor inhibition: conjunctive brain activations across different versions of go/no-go and stop tasks. NeuroImage, 13, 250-261.

Sauseng, P., \& Klimesch, W. (2008). What does phase information of oscillatory brain activity tell us about cognitive processes? Neuroscience and Biobehavioral Reviews, 32, 1001-1013. doi:10.1016/j.neubiorev.2008.03.014.

Savostyanov, A. N., Tsai, A. C., Liou, M., Levin, E. A., Lee, J. D., Yurganov, A. V., et al. (2009). EEG-correlates of trait anxiety in the stop-signal paradigm. Neuroscience Letters, 449, 112-116. doi:10.1016/j.neulet.2008.10.084.

Shackman, A. J., McMenamin, B. W., Maxwell, J. S., Greischar, L. L., \& Davidson, R. J. (2009). Right dorsolateral prefrontal cortical activity and behavioral inhibition. Psychological Science, 20, 1500-1506.

Sharp, D. J., Bonnelle, V., De Boissezon, X., Beckmann, C. F., James, S. G., Patel, M. C., et al. (2010). Distinct frontal systems for response inhibition, attentional capture, and error processing. Proceedings of the National Academy of Sciences, 107, 61066111. doi:10.1073/pnas.1000175107.

Snedecor, G. W., \& Cochran, W. G. (1967). Statistical methods (6th ed.). Ames: Iowa State University Press.

Wacker, J., Chavanon, M. L., Leue, A., \& Stemmler, G. (2010). Trait BIS predicts alpha asymmetry and p300 in a go/no-go task. European Journal of Personality, 24, 85-105.

Yeung, N., Botvinick, M. M., \& Cohen, J. D. (2004). The neural basis of error detection: conflict monitoring and the error-related negativity. Psychological Review, 111, 931-959. doi:10.1037/ 0033-295x.111.4.931.

Young, C. K., \& McNaughton, N. (2008). Coupling of theta oscillations between anterior and posterior midline cortex and with the hippocampus in freely behaving rats. Cerebral Cortex, 19, 24-40. 